\title{
Serrated plastic flow
}

\section{P RODRIGUEZ}

Metallurgy Programme, Reactor Research Centre, Kalpakkam 603 102, India

\begin{abstract}
This paper attempts an assessment of the current understanding of the phenomenon of "serrated plastic flow", which manifests itself as serrations, load drops, jerkiness or other discontinuities in the stress-strain curves obtained in constant extension rate tensile tests, and as sudden bursts of strain in constant loading rate tests and in constant load (stress) creep tests (the so called staircase creep). Though one can identify at least seven physical processes that can cause serrations, the discussion here is restricted mainly to serrated yielding in tension tests originating from dynamic strain ageing (DSA). The characteristics of the five types of serrations that have been identified so far and the experimental conditions under which they occur are discussed. The various models of serrated flow that have been put forward are reviewed critically. Some recent results on 316 stainless steel are presented to illustrate the effects of grain size, temperature and strain rate on serrated flow. Manifestations of DSA other than serrations such as a negative strain rate sensitivity, positive temperature dependence for flow stress and work hardening, and the ductility minimum are also discussed. Finally the various issues to be resolved are enumerated.
\end{abstract}

Keywords. Serrated flow; dynamic strain ageing; continual mechanical twinning; flow stress; work hardening peaks; ductility minimum.

\section{Introduction}

The organisers of this discussion meeting in their invitation requested that I should present here the metallurgist's viewpoint of 'serrated plastic flow'. At the outset let me state that whatever I say in the following should be considered as my own understanding of the phenomenon. There will be many materials scientists and engineers (including those with both physics and metallurgy backgrounds) who may not agree with all the views propounded here; there must also be an equally large number of physicists and metallurgists who may find common ground with the interpretations offered. It is interesting to note that in a recent international conference on deformation, two different treatments of the phenomenon, both by metallurgists have been given. One is based on a strain ageing mechanism involving the diffusion of solute atoms to temporarily arrested dislocations (McCormick 1983) and the other interprets it as an example of catastrophe theory in physical metallurgy (Strudel 1983).

The objective of the present paper is an assessment of the current understanding of the phenomenon of serrated plastic flow. We start with a brief description of the methodology of the tensile test. The physical processes that can cause serrated flow are then identified. Subsequent discussions are restricted to serrations due to dynamic strain ageing (DSA) or the Portevin-Le Chatelier (PL) effect (Portevin and Le Chatelier 1923) arising from interactions between diffusing solute atoms and mobile dislocations. The characteristics of five types of serrations observed under conditions of DSA are then discussed. The various quantitative models for serrated flow that have been put forward are then critically reviewed. Some recent results on 316 stainless steel are presented to 
illustrate the effects of grain size, temperature and strain rate on the DSA behaviour. This is followed by a discussion of the various manifestations of DSA in tensile test results. Finally, the various issues to be resolved are considered and catalogued.

\section{Phenomenology}

In a tension test, (the specimen suitably gripped between two crossheads, one moving and the other fixed) is deformed at a constant nominal strain rate determined by the velocity of the moving crosshead. At any instant of time, the total strain $\varepsilon$, the plastic strain $\varepsilon_{p}$ in the specimen, and the elastic strain $\varepsilon_{e}$ of the specimen-machine system are related by

$$
\begin{aligned}
& \varepsilon=\varepsilon_{p}+\varepsilon_{e}, \\
& \dot{\varepsilon}=\dot{\varepsilon}_{p}+\dot{\varepsilon}_{e} .
\end{aligned}
$$

Equation (2) can be rewritten as

$$
\dot{\varepsilon}=\dot{\varepsilon}_{p}+\frac{1}{E_{s}} \dot{\sigma},
$$

where $\sigma$ is the stress and $E_{s}$ the elastic modulus of the specimen-machine system. The condition for serrations or load drops is that the plastic strain rate $\dot{\varepsilon}_{p}$ exceeds the imposed strain rate $\dot{\varepsilon}$, i.e. whenever there is a sudden increase in $\dot{\varepsilon}_{p}$, a load drop occurs.

The phenomenon can also occur in creep tests in which strain in a specimen under a constant stress is measured as a function of time. Sudden bursts of plastic strain occur periodically, with the result that the creep curve has the shape of a staircase (hence the name 'staircase creep'). This manifestation of DSA in creep tests is discussed in another paper (Ananthakrishna 1983) presented at this Meeting.

\section{Physical processes that can cause serrated flow}

A survey of the literature shows that we can identify at least seven physical processes that can lead to serrations in the flow curve:

(i) The plastic strain rate under dislocation glide is given by

$$
\dot{\varepsilon}_{p}=\rho_{m} b \bar{v},
$$

where $\rho_{m}$ is the mobile dislocation density, $\bar{v}$ the average velocity of dislocations and $b$ the Burgers vector. Serrations will therefore occur whenever there is an instantaneous increase in $\rho_{m}$ or in $\bar{v}$ or in both.

(ii) Interaction of moving dislocations with DSA can lead to sudden increases in $\rho_{m}$ and/or $\bar{v}$ and we shall be mainly examining this particular phenomenon.

(iii) In alloys undergoing order-disorder transformations, gradients or modulations in order encountered by moving dislocations can cause serrated flow (Mannan and Rodriguez 1972; Samuel and Rodriguez 1975, 1980).

(iv) Another phenomenon that can cause serrations is continual mechanical twinning as has been reported for an Fe-25 at \% Be alloy (Bolling and Richman 1965). Twinning is characterised by a positive temperature dependence and a negative strain rate sensitivity for the flow stress which are also obtained under conditions of DSA. 
(v) A sudden increase in the specimen temperature due to adiabatic heating is another possibility (Ramachandran 1983; Ramachandran et al 1970; Kubin et al 1982). This has been found to occur in tests at cryogenic temperatures. Dissipiation of the heat of deformation becomes difficult since the specimen surface is covered by a layer of gas molecules of the cryogenic liquid. The resultant increase in specimen temperature satisfies the condition for a load drop since a higher plastic strain rate can now occur at the applied stress level.

(vi) Phase transformations induced by stress and strain can also cause serrated flow. This aspect is covered in another paper presented at this Meeting (Seetharaman 1983).

(vii) Weertman (1967) has pointed out that yielding across fracture surfaces in brittle materials when tested under both hydrostatic pressure and triaxial non-hydrostatic stresses can cause serrations.

\section{Types of serrations}

Five types of serrations due to DSA termed A, B, C, D and E have so far been identified (figure 1). Types A, B and C are well-known and their characteristics are also well documented (Russel 1963; Macherauch and Munz 1966; Solar-Gomez and McG-Tegart 1969; Brindley and Worthington 1970; Cuddy and Leslie 1972) whereas serrations of types $D$ and $E$ have been referred to only relatively recently (Pink and Grinberg 1981; Wijler et al 1972). The characteristics of the different types of serrations and the experimental conditions that produce them are as follows.

(i) Type A serrations are periodic serrations from repeated deformation bands initiating at the same end of the specimen and propagating in the same direction along the gauge length. These are considered as locking serrations, characterised by an abrupt rise followed by a drop to below the general level of the stress-strain curve. They occur in the low temperature (high strain rate) part of the DSA regime.

(ii) Type B serrations are oscillations about the general level of the stress-strain curve that occur in quick succession due to discontinuous band propagation arising from the DSA of the moving dislocations within the band. (This contrasts with the smooth propagation of the band that occurs after initiation with a type A load drop). Type B

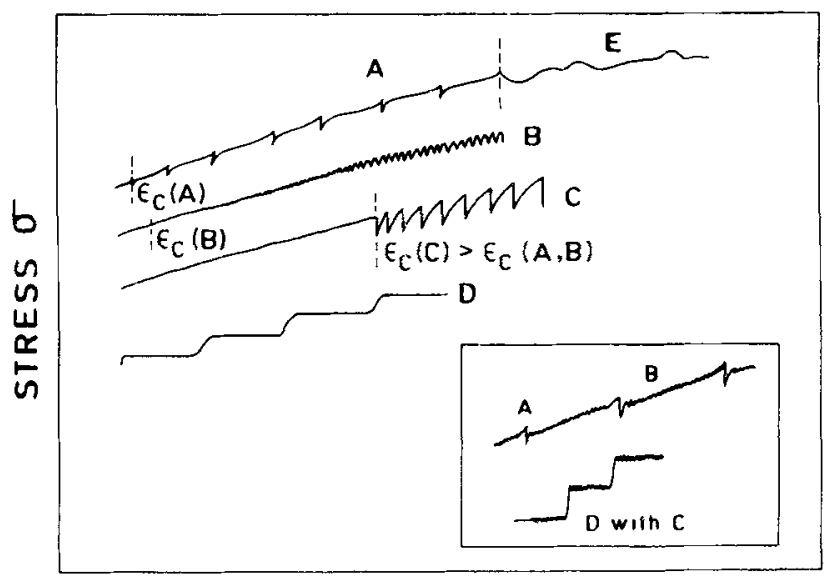

Figure 1. Types of serrations.

STRAIN E 
serrations often develop from those of type A with increasing strain (figure 1 inset) or occur at the onset of serrated yielding at high temperatures and low strain rates.

(iii) Type $C$ serrations are yield drops that occur below the general level of the flow curve and are therefore considered to be due to dislocation unlocking. They occur at higher temperatures and lower strain rates than in the case of types A and B serrations.

(iv) Type $\mathrm{D}$ serrations are plateaus in the $\sigma-\varepsilon$ curve due to band propagation similar to Luders band with no work hardening or strain gradient ahead of the moving band front. Like type A serrations, they can occur alone or with type B serrations during the band propagation (figure 1 inset). In $\mathrm{Au}-\mathrm{Cu} 14 \mathrm{wt} \%$ alloy, Wijler et al (1972) produced type D serrations by homogeneous prestraining at high temperatures.

(v) Type A serrations change over to ty pe E serrations at high strains. The latter are similar to type A serrations, but with little or no work hardening during band propagation.

\section{Models for serrated flow from DSA}

Any theory or model for serrated flow should account for the different types of serrations that appear in different alloys and for the influence of temperature, strain and strain rate on these various types. For the onset of serrations in substitutional alloys, a critical strain $\varepsilon_{c}$ is required. The $\varepsilon_{c}$ value is dependent on both $\dot{\varepsilon}$ and the temperature $T$ (figure 2). At high strain rates and low temperatures where the first serrations are of type $\mathrm{A}$ or $\mathrm{B}, \varepsilon_{\mathrm{c}}$ increases with increasing strain rate and decreasing temperature. At high temperatures and low strain rates, where serrations of type $C$ are observed, $\varepsilon_{c}$ increases with increasing temperature and decreasing strain rate. While current theoretical models are unable to account for this latter behaviour, the variation of $\varepsilon_{c}$ with $T$ and $\dot{\varepsilon}$ in the low temperature (high strain rate) regime has been rationalized by a number of theoretical models based on the interaction of diffusing solute atoms and mobile dislocations.

\subsection{Interpretations of $\varepsilon_{c}$}

The oldest quantitative model for serrated yielding is due to Cottrell (1953) in which the mobile solutes are assumed to interact with quasi-viscously moving dislocations; serrations are attributed to repeated locking and unlocking of dislocations in solute atmospheres, which occurs when $\bar{v}$ equals the drift velocity of the solutes in the stress field of the dislocations. In subsequent modifications of this model that integrate it with the theory of thermally activated deformation processes, it was recognised (Sleeswyk

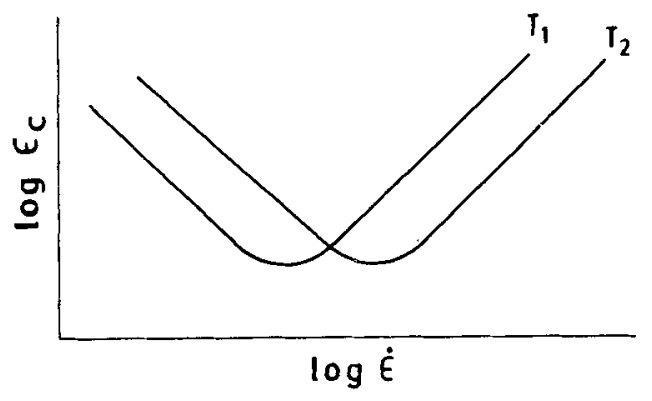

Figure 2. Effect of strain rate and temperature on critical strain; $T_{2}>T_{1}$. 
1958; McCormick 1972) that the "ageing" of the dislocations would take place during the waiting time of the dislocations at discrete obstacles rather than during their actual motion. When the diffusion coefficient is high enough to saturate the dislocation with a solute atmosphere during the waiting time, serrated yielding will start. In the model of van den Beukel (1975) though the same picture of solute-dislocation interaction was adopted, $\varepsilon_{c}$ was chosen (following the ideas of Sleeswyk 1958; Penning 1972) to be the strain at which the strain rate sensitivity $\gamma=\partial \sigma / \partial \ln \dot{\varepsilon}$ became negative. In all the three approaches, the final result is that at $\varepsilon_{c}$

$$
\rho_{m}^{(c)} C_{v}^{(c)}=K_{1} \dot{\varepsilon} \exp \left(E_{m} / k T\right),
$$

where $C_{v}$ is the vacancy concentration $E_{m}$ is the vacancy migration energy and $T$ the absolute temperature. The three models yield widely different values for the constant $K_{1}$ (the value in the original Cottrell model does not account for the experimental values of $\varepsilon_{c}$ ). The vacancy concentration is enhanced due to the strain (Van Bueren 1955) according to

$$
C_{v}=A \varepsilon^{m} \text {. }
$$

Similarly, the strain dependence of $\rho_{m}$ can be expressed as

$$
\rho_{m}=B \varepsilon^{\beta} \text {. }
$$

From (5), (6) and (7) we get

$$
\varepsilon_{c}^{m+\beta}=K_{2} \dot{\varepsilon} \exp \left(E_{m} / k T\right)
$$

DSA due to interstitial solutes (8) reduces to

$$
\varepsilon_{c}^{\beta}=K_{2}^{\prime} \dot{\varepsilon} \exp \left(E_{m} / k T\right)
$$

Experimental results in the low temperature-high strain rate region have given reasonable values for $m, \beta$ and $E_{m}$. Typical $(m+\beta)$ values are between 2 and 3 for substitutional alloys; for interstitial alloys (Van den Beukel 1980), $\beta$ lies between 0.5 and 1.

It has generally been observed that the critical strain $\varepsilon_{c}$ increases with increasing grain size (Brindley and Worthington 1970; Charnock 1968, 1969; Mannan 1981; Mannan et al 1983). Relating this increase to the grain size dependence of $\rho_{m}$, Charnock (1968, 1969) has shown that (8) can be modified to include the grain size dependence of $\varepsilon_{c}$ according to

$$
\varepsilon_{c}=K_{3} d^{p} \varepsilon^{q} \exp \left[E_{m} / k T(m+\beta)\right]
$$

where $p=n^{\prime \prime} /(m+\beta), q=1 /(m+\beta)$ and the parameter $n^{\prime \prime}$ arises from the $\rho_{m}-d$ relationship of Conrad and Christ (1963): $\rho_{m}=N \varepsilon^{\beta} / d^{n^{\prime \prime}}$.

\subsection{Some results on 316 stainless steel}

The flow curves obtained in the temperature range $523-923 \mathrm{~K}$ at a strain rate of 3 $\times 10^{-4} \mathrm{sec}^{-1}$ for 316 stainless steel of two different grain sizes $(0.04$ and $0.125 \mathrm{~mm})$ are shown in figures 3 and 4 (Mannan 1981; Mannan et al 1982, 1983). (The serration types are labelled as in \$4). The observed effects of the grain size on DSA are the following: (a) The DSA temperature region gets shifted to higher temperatures with increasing grain size; (b) For all grain sizes (8) is valid with $(m+\beta)=2 \cdot 3, \beta$ was estimated to be $\simeq 0 \cdot 9$, in 


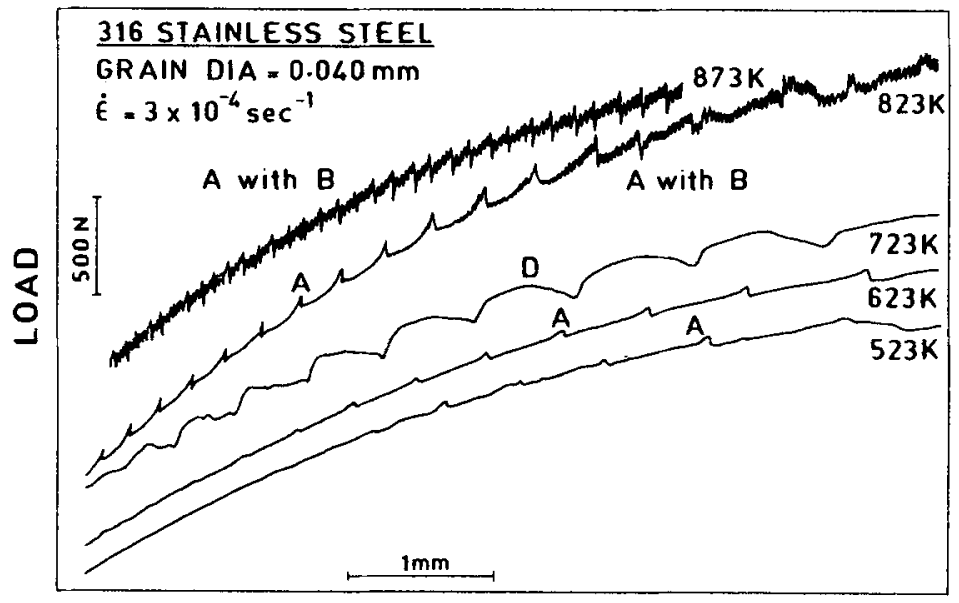

ELONGATION

Figure 3. Serrated plastic flow in 316 stainless steel with grain size $d=0.04 \mathrm{~mm}$.

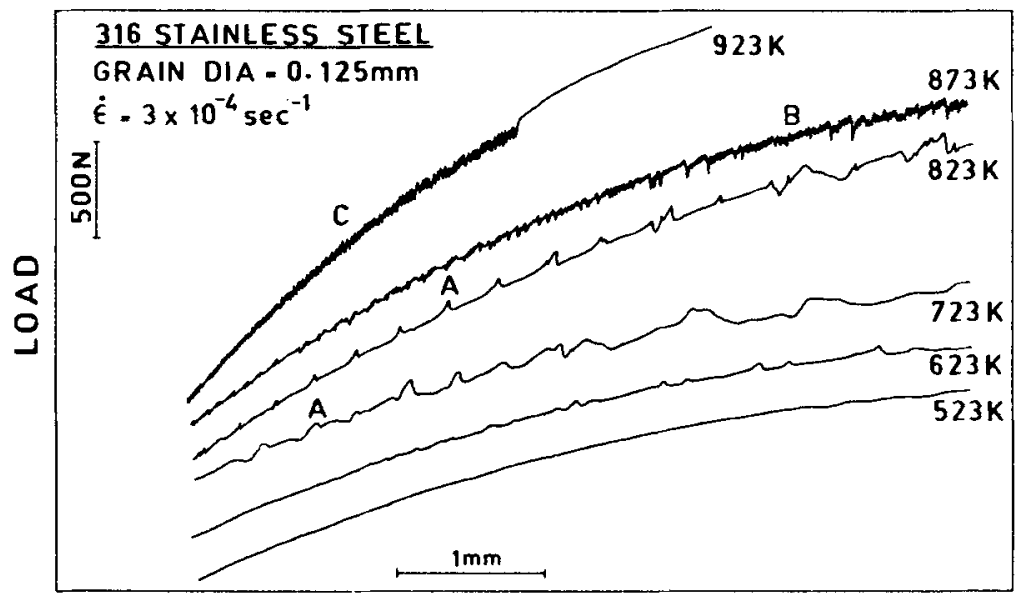

ELONGATION

Figure 4. Serrated plastic flow in 316 stainless steel with grain size $d=0.125 \mathrm{~mm}$.

good agreement with the prediction of $\beta=1$ for a linear increase in dislocation density with strain; (c) The relationship between $\varepsilon_{c}$ and $d$ follows (10) with $p=0.6$; (d) An apparent activation energy for DSA of $255 \mathrm{~kJ} / \mathrm{mol}$ is obtained for all grain sizes from the variation of $\varepsilon_{c}$ with $T$, (8); (e) A fit to the Hall-Petch equation for the variation of the flow stress $\sigma_{\varepsilon}$ with grain size $d$, namely, $\sigma_{\varepsilon}=\sigma_{0 z}+K_{\varepsilon} d^{-1 / 2}$ where $K_{\varepsilon}$ and $\sigma_{0 \varepsilon}$ are constants (called the Hall-Petch slope and intercept respectively), yields a monotonic decrease of $\sigma_{0 \varepsilon}$ with temperature; whereas $K_{\varepsilon}$ though it generally decreases with temperature, shows a peak in the temperature region $523-723 \mathrm{~K}$. This indicates that grain boundary regions are preferred sites for dynamic strain ageing (Armstrong 1968); (f) A peak in the variation of work hardening with temperature is also obtained but towards the upper 


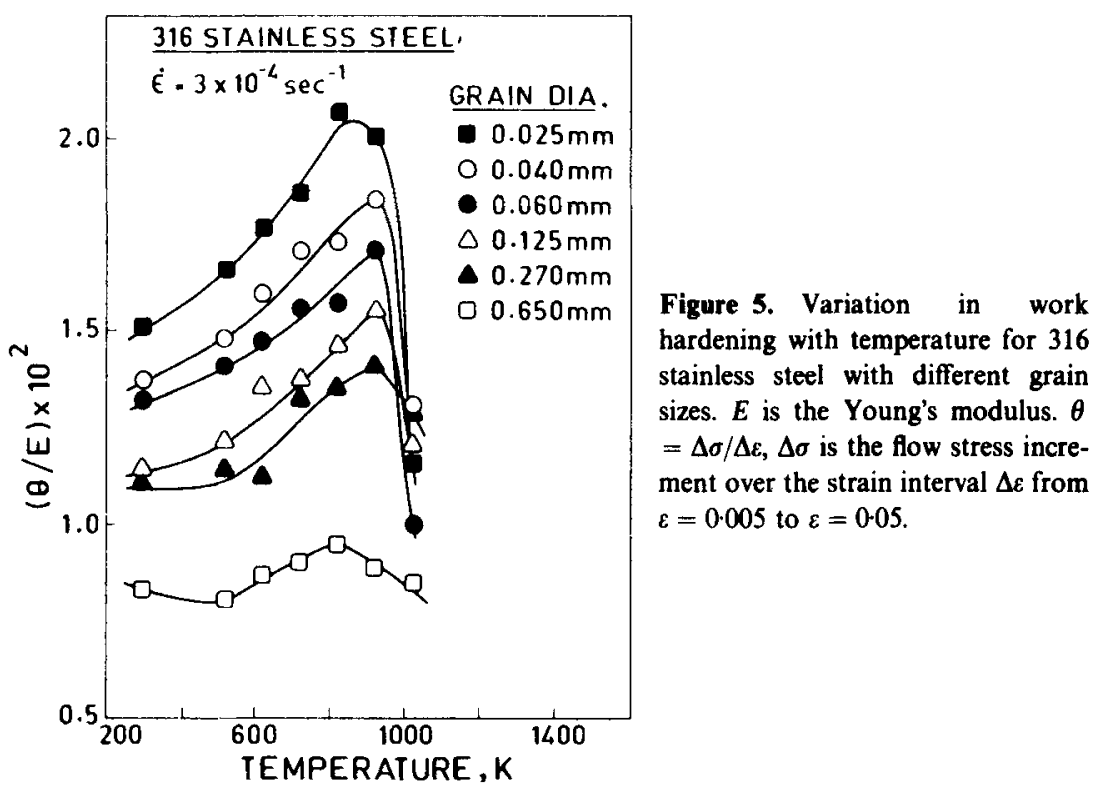

end of the DSA temperature range (figure 5). Consistent with the fact that this temperature range represents conditions for strong pinning and accordingly the most rapid and strongest dislocation immobilisation, leading to an increased rate of dislocation accumulation (Reed-Hill 1974), the DSA peak for work hardening also becomes more pronounced with decreasing grain size.

\subsection{Other interpretations of serrated flow}

Equation (4) allows serrations to occur for sudden increases in $\rho_{m}$ or in $\bar{v}$ or in both of them. The models discussed in $\$ 5 \cdot 1$ assume that the average velocity is unchanged, and that serrations occur due to changes in $\rho_{m}$ owing to the locking and unlocking of dislocations during deformation. It has also been pointed out that the solute atmospheres formed could be dragged along with the moving dislocations, and that such atmospheres would exert a drag stress on the dislocation which varies with dislocation velocity as schematically illustrated in figure 6(a) (Hirth and Lothe 1968; Schmidt and Miller 1982). With this drag stress contribution included, the normal stress-velocity variation for dislocations shown in figure 6(b) assumes the shape shown in figure 6(c). Nabarro (1967) has suggested that serrations will occur in the strain rate (dislocation velocity) region between the points $B$ and $D$ in this figure since the dislocation velocity is a multivalued function of stress in this region; for a given value of the applied stress it is possible for the velocity to increase suddenly to a higher value. The region between $\mathrm{C}$ and $\mathrm{D}$ where the moving dislocation requires a lower stress for $\mathrm{a}$ higher velocity (negative strain rate sensitivity) is more commonly identified with the dynamic instability of plasticity leading to the PL effect (Sleeswyk 1958; Sleeswyk and Verel 1972; Wilcox and Rosenfield 1966; Yoshinaga and Morozumi 1971; Penning 1972; Malygin 1973). Suggestions have also been made that serrated yielding could be considered as a manifestation of 'chaotic' behaviour (Neelakantan and Venkataraman 1982) or as an example of a 'catastrophe' in plastic deformation (Strudel 1983) arising from the negative-resistance feature between $C$ and $D$. 


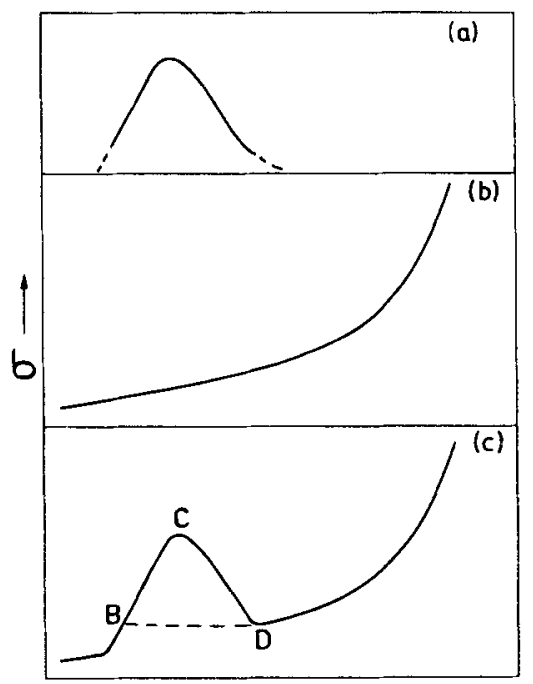

$\log \bar{v} \longrightarrow$
Figure 6. (a) The solute drag stress on a dislocation as a function of dislocation velocity. (b) Normal stress-velocity relation for dislocation in the absence of the drag stress. (c) Actual stress-velocity variation due to the presence of a drag stress at intermediate velocities.

That negative strain rate sensitivity is a crucial factor for the PL effect is now recognised in all the models. The treatment of McCormick (1983), for example, points out that the critical strain for the onset of serrated flow can be rationalised by matching the critical velocity (for negative resistance) with the solute diffusion coefficient. In the models of Van den Beukel $(1975,1980)$ and of Mulford and Kocks (1979) it is held that any solute mobility makes a negative contribution to the total strain rate sensitivity and that this contribution increases with strain; when the total strain rate sensitivity becomes negative plastic flow becomes unstable. In arriving at the strain dependence of the strain rate sensitivity, Van den Beukel retains the notions of a strain-dependent solute diffusion coefficient and strain-dependent mobile dislocation density from the older models. Mulford and Kocks (1979), on the other hand, describe the strain rate sensitivity in terms of the flow stress $\sigma$ which is the sum of two components--the friction stress $\sigma_{f}$ and the dislocation flow stress $\sigma_{d}$; solute mobility affects $\sigma_{d}$, the strainhardening component of the flow stress. More recently, Van den Beukel and Kocks (1982) have arrived at a unified approach which enables the negative strain rate sensitivity to arise as a consequence of the influence of solute mobility on both the friction stress (by decreasing the obstacle spacing along the dislocation) and forest hardening (by increasing the strength of dislocation junctions).

In a paper that has not received the attention that it deserves, Weertman (1967) has suggested that the pL effect occurs as a result of an instability of the dislocations in going back and forth between a slow moving state and a fast moving state due to dynamic frictional stress drops. The effect occurs because of the inherent instability of the position that separates the fast slip region from the slow slip region in the slipped zone. The similarity between earthquakes and the behaviour of groups of dislocations in a slip band when the frictional force is reduced for any reason has also been pointed out: "Earthquakes are merely, the PL effect exhibited in a sample whose dimensions happen to have a grand scale".

Mention should also be made of attempts to understand serrated yielding through mechanical and electronic models. Dry friction models that simulate the strain bursts in 
staircase creep (Bodner and Rosen 1967) and the load drops in tensile tests (Rosen and Bodner 1969) have been devised which consider some of the features of DSA such as the negative strain rate sensitivity, work hardening and deformation by band propagation. Similar electronic analogs for the tensile test situation have also been demonstrated (Neelakantan and Venkataraman 1983).

\section{Other manifestations of DSA}

As we have mentioned earlier, serrations represent only one of the manifestations of dynamic strain ageing. In figure 7 the various other anomalies associated with DSA are illustrated schematically. These are (i) a peak in the variation of $\sigma$ with $T$ (ii) a peak in the variation of the work hardening $\theta=\Delta \sigma / \Delta \varepsilon$ with $T$, (iii) a peak in the variation of the Hall-Petch slope $K_{\varepsilon}$ with $T$, (iv) a minimum in the variation of the ductility with $T$ and (v) a minimum in the strain rate sensitivity $\gamma=\Delta \sigma / \Delta \ln \dot{\varepsilon}$ with $\gamma$ going negative in the temperature region of serrated flow. The variations in $\gamma$ with stress and strain at different temperatures are also shown in figure 7. The types of serrations normally observed in the different temperature regions are also indicated.

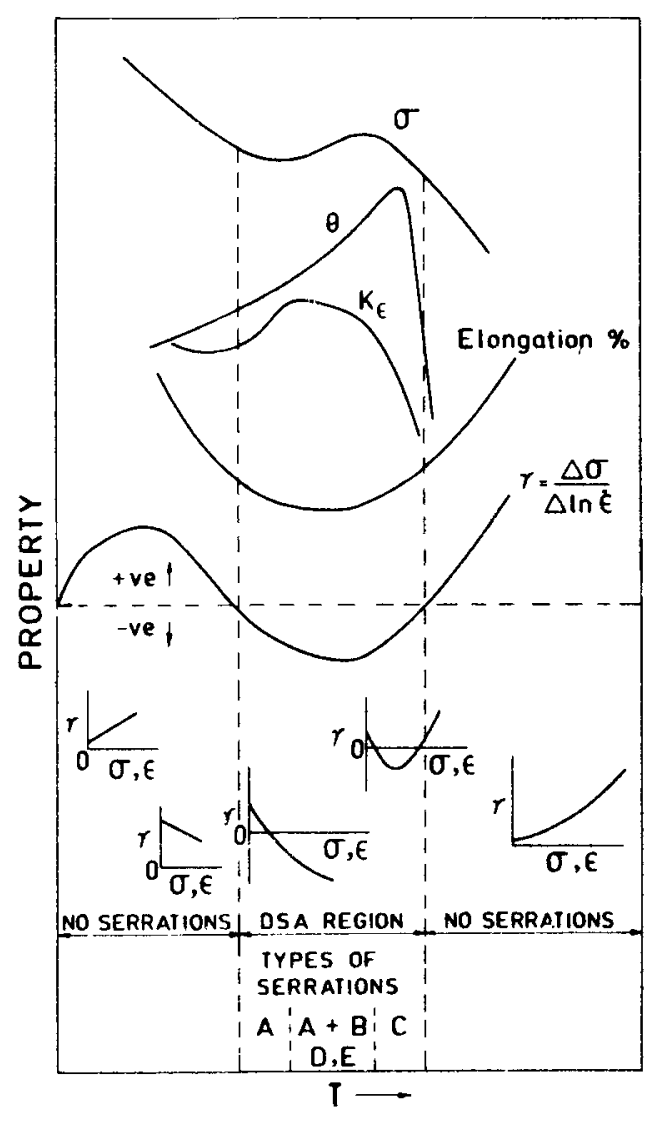

Figure 7. Schematic illustration of the various manifestations of dynamic strain ageing. 


\section{Issues to be resolved}

(i) The main issue to be resolved unambiguously is whether the load drops are caused by a sudden increase in $\rho_{m}$ or in $\bar{v}$ or in both. We are handicapped by the fact that we do not so far have an experimental technique to measure $\rho_{m}$ or $\bar{v}$ during deformation. Acoustic emission measurements hold out promise as a potential method of attack on this problem.

(ii) Deformation during serrated flow is inhomogeneous and localised in bands. The actual strain rate in the deformation band is much higher than the imposed or assumed strain rate. Measurements and analysis of deformation band parameters such as the band velocity, band strain, band width and the average strain rate in the band should therefore receive greater attention than they have hitherto (McCormick 1983).

(iii) Current theoretical models are unable to account for the inverse effects $\left(\varepsilon_{c}\right.$ increasing with increasing temperature and decreasing strain rate) observed in the high temperature-low strain rate region in which type $\mathrm{C}$ serrations are observed.

(iv) Most of the models are incomplete in that they make ad hoc assumptions or use empirical relationships; there is no detailed understanding either experimentally or theoretically of the effect of strain on diffusion, of the dependence of mobile dislocation density on strain or of the effect of a solute atmosphere on dislocation-dislocation interactions.

(v) Though it is often recognised that only short range diffusion in dislocation core (pipe diffusion) is involved in many manifestations of DSA, we are handicapped by the lack of an adequate knowledge of the appropriate diffusion coefficients.

(vi) In analysing the dependence of $\sigma$ on $\dot{\varepsilon}$ and $T$, is the splitting of the flow stress into additive contributions from forest interactions, solutes, lattice friction, justified? How is such an approach reconciled with the idea that the obstacle with the highest energy barrier will be rate-controlling?

This list is evidently not an exhaustive one, but it does highlight some of the aspects of DSA that deserve greater attention from both experiment and theory in future.

\section{References}

Ananthakrishna G 1984 Bull. Mater. Sci.

Armstrong R W 1968 in Dislocation dynamics (eds) A R Rosenfield, G T Hahn, A L Bement and R I Jaffee (New York: McGraw Hill) p. 293

Bodner S R and Rosen A 1967 J. Mech. Phys. Solids 1563

Brindley B J and Worthington P J 1970 Met. Rev. 15101

Bolling G F and Richman R H 1965 Acta Metall. 13 709, 723, 745

Charnock W 1968 Philos. Mag. 1889

Charnock W 1969 Philos. Mag 20209

Conrad H and Christ B 1963 in Recovery and recrystallization of metals (ed) L Himmel (New York: Interscience) p. 124

Cottrell A H 1953 Philos. Mag 44829

Cuddy L J and Leslie W C 1972 Acta Metall. 201157

Hirth J P and Lothe J 1968 Theory of crystal dislocations (New York: McGraw-Hill)

Kubin L P, Spiesser Ph and Estrin Y 1982 Acta Metall. 30385

Macherauch E and Munz D 1966 Z. Metall. 57552

Malygin G A 1973 Phys Status Solidi A15 51

Mannan S L 1981 Infuence of grain size on the flow and fracture in AISI type 316 stainless steel at elevated temperatures Ph.D. Thesis, Indian Institute of Science, Bangalore 
Mannan S L and Rodriguez P 1972 Philos. Mag. 25673

Mannan S L, Samuel K G and Rodriguez P 1982 in Proc. 6th Int. Conf. Strength of Metals and Alloys, Melbourne, Aug. 1982 (ed) R C Giffkins (New York: Pergamon Press) Vol 2 pp. 637-642

Mannan S L, Samuel K G and Rodriguez P 1983 Trans. Indian Inst. Metals 36313

McCormick P G 1972 Acta Metall. 20351

McCormick P G 1983 in Deformation-All aspects, Int. Conf. on Metal Sci., ICMS, Ranchi (Calcutta: Indian Institute of Metals)

Mulford R A and Kocks U F 1979 Acta Metall. 271125

Nabarro F R N 1967 Theory of crystal dislocation (Oxford: University Press) p. 431

Neelakantan K and Venkataraman G 1983 Acta Metall. 3177

Penning P 1972 Acta Metall. 201169

Pink E and Grinberg A 1981 Mater. Sci. Eng. 511

Portevin A and LeChatelier F 1923 CR Acad. Sci. 176507

Ramachandran V 1983 in Deformation-All aspects, Int. Conf. on Metal Sci., ICMS, Ranchi (Calcutta: Indian Institute of Metals)

Ramachandran V, Baldwin D H and Reed-Hill R E 1970 Metall. Trans. 13011

Reed-Hill R E 1974 Rev. High Temp. Mater. 2214

Rosen A and Bodner S R 1969 Mater. Sci. Eng. 4115

Russel B 1963 Philos. Mag. 8615

Samuel K G and Rodriguez P 1975 Trans. Indian Inst. Metals 28323

Samuel K G and Rodriguez P 1980 Trans Indian Inst. Metals 33285

Schmidt C G and Miller A K 1982 Acta Metall. 30615

Seetharaman V 1984 Bull Mater. Sci. 6

Sleeswyk A W 1958 Acta Metall. 6598

Sleeswyk A W and Verel D J 1972 Scr. Metall. 61972

Solar-Gomez A J R and McG Tegart W J 1969 Philos. Mag. 20507

Strudel J L 1983 in Deformation-All Aspects, Int. Conf. on Metal Sci., ICMS, Ranchi, (Calcutta: Indian Institute of Metals)

Van Bueren H G 1955 Acta Metall. 3519

Van den Beukel A 1975 Phys Status Solidi A30 197

Van den Beukel A 1980 Acta Metall. 28965

Van den Beukel A and Kocks U F 1982 Acta Metall. 301027

Weertman J 1967 Can. J. Phys. 45797

Wijler A, Schade Van Westrum J and Van den Beukel A 1972 Acta Metall. 20355

Wilcox B A and Rosenfield A R 1966 Mater. Sci. Eng. 1201

Yoshinaga H and Morozumi S 1971 Philos. Mag. 23 1351, 1367 